Acta Crystallographica Section E

Structure Reports

Online

ISSN 1600-5368

\section{2,3-Bis(methylsulfanyl)-1,4,5,8-tetrathia- fulvalene}

\section{Ning-Juan Zheng and Bing-Zhu Yin*}

Key Laboratory of Organism Functional Factors of the Changbai Mountain, Yanbian University, Ministry of Education, Yanji 133002, People's Republic of China

Correspondence e-mail: zqcong@ybu.edu.cn

Received 19 November 2010; accepted 22 November 2010

Key indicators: single-crystal X-ray study; $T=291 \mathrm{~K}$; mean $\sigma(\mathrm{C}-\mathrm{C})=0.003 \AA$; $R$ factor $=0.031 ; w R$ factor $=0.083 ;$ data-to-parameter ratio $=21.5$.

In the title compound, $\mathrm{C}_{8} \mathrm{H}_{8} \mathrm{~S}_{6}$, the five-membered rings form a dihedral angle of $25.06(9)^{\circ}$. In the absence of short intermolecular contacts, the molecules are packed by van der Waals forces in the crystal.

\section{Related literature}

For applications of tetrathiafulvalenes, see: Wudl et al. (1972); Jørgensen et al. (1994). For details of the synthesis, see: Fourmingué et al. (1993). For a related structure, see: Hou et al. (2010).

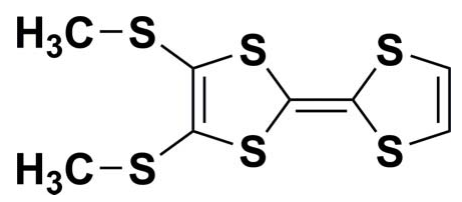

\section{Experimental}

\section{Crystal data}

$\mathrm{C}_{8} \mathrm{H}_{8} \mathrm{~S}_{6}$

Monoclinic, $C 2 / c$

$a=19.368$ (11) ̊
$M_{r}=296.50$

$$
Z=8
$$

Mo $K \alpha$ radiation

$\mu=1.09 \mathrm{~mm}^{-1}$

Data collection

Rigaku R-AXIS RAPID diffractometer

Absorption correction: multi-scan (ABSCOR; Higashi, 1995)

$T_{\min }=0.881, T_{\max }=0.909$

\section{Refinement}

$R\left[F^{2}>2 \sigma\left(F^{2}\right)\right]=0.031$

$w R\left(F^{2}\right)=0.083$

$S=1.03$

2773 reflections
$T=291 \mathrm{~K}$

$0.12 \times 0.10 \times 0.09 \mathrm{~mm}$

11301 measured reflections 2773 independent reflections 2480 reflections with $I>2 \sigma(I)$ $R_{\text {int }}=0.025$
Data collection: RAPID-AUTO (Rigaku, 1998); cell refinement: RAPID-AUTO; data reduction: CrystalStructure (Rigaku/MSC, 2002); $\operatorname{program}(\mathrm{s})$ used to solve structure: SHELXS97 (Sheldrick, 2008); program(s) used to refine structure: SHELXL97 (Sheldrick, 2008); molecular graphics: PLATON (Spek, 2009); software used to prepare material for publication: SHELXL97.

The authors acknowledge financial support from the National Natural Science Foundation of China (grant No. 20662010), the Specialized Research Fund for the Doctoral Programme of Higher Education (grant No. 2006184001) and the Open Project of the State Key Laboratory of Supramolecular Structure and Materials, Jilin University.

Supplementary data and figures for this paper are available from the IUCr electronic archives (Reference: NG5069).

\section{References}

Fourmingué, M., Krebs, F. C. \& Larsen, J. (1993). Synthesis, 5, 509-512. Higashi, T. (1995). ABSCOR. Rigaku Corporation, Tokyo, Japan. Hou, R.-B., Li, B., Yin, B.-Z. \& Wu, L.-X. (2010). Acta Cryst. E66, o1044. Jørgensen, T., Hansen, T. K. \& Becher, J. (1994). Chem. Soc. Rev. 23, 41-45. Rigaku (1998). RAPID-AUTO. Rigaku Corporation, Tokyo, Japan.

Rigaku/MSC (2002). CrystalStructure. Rigaku/MSC Inc., The Woodlands, Texas, USA.

Sheldrick, G. M. (2008). Acta Cryst. A64, 112-122.

Spek, A. L. (2009). Acta Cryst. D65, 148-155.

Wudl, F., Wobshall, D. \& Hufnagel, E. J. (1972). J. Am. Chem. Soc. 94, 670-672. $c=17.150(8) \AA$

$\beta=108.59$ (2) ${ }^{\circ}$

$V=2425$ (2) $\AA^{3}$ $b=7.703$ (4) $\AA$ 


\section{supporting information}

Acta Cryst. (2010). E66, o3306 [https://doi.org/10.1107/S1600536810048580]

\section{2,3-Bis(methylsulfanyl)-1,4,5,8-tetrathiafulvalene}

\section{Ning-Juan Zheng and Bing-Zhu Yin}

\section{S1. Comment}

Tetrathiafulvalenes (TTFs) have attracted much interest due to their electron-donating ability, which have been used for the synthesis of new organic metals and superconductors (Wudl et al. 1972) and recently for supramolecular architectures (Jørgensen et al. 1994.). In this paper, we report the crystal structue of the title compound.

The title compound, as shown in Fig. 1, crystallizes in monoclinic system with the space group $C 2 / c$. All bond lengths and angles of the title compound are normal and comparable with those reported for the related structure (Hou et al., 2010). In the crystal, the molecules are packed by van der Waal's forces.

\section{S2. Experimental}

The title compound was prepared according to literature (Fourmingué et al., 1993) and single crystals suitable for X-ray diffraction were prepared by slow evaporation a mixture of dichloromethane and petroleum $\left(60-90{ }^{\circ} \mathrm{C}\right)$ at room temperature.

\section{S3. Refinement}

Carbon-bound $\mathrm{H}$-atoms were placed in calculated positions with $\mathrm{C}-\mathrm{H}=0.93$ or $0.96 \AA$ and were included in the refinement in the riding model with $U_{\text {iso }}(\mathrm{H})=1.2$ or $1.5 U_{\mathrm{eq}}(\mathrm{C})$.

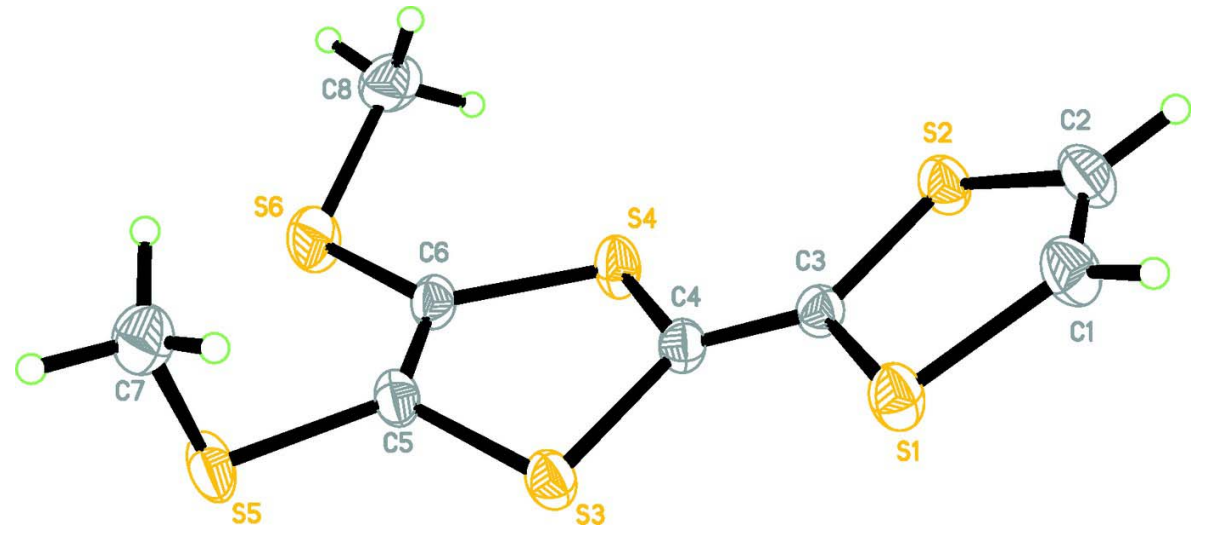

Figure 1

The asymmetric of title compound, with the atom numbering. Displacement ellipsoids of non-H atoms are drawn at the $30 \%$ probalility level. 
2-(2H-1,3-dithiol-2-ylidene)-4,5-bis(methylsulfanyl)-2H-1,3-dithiole

Crystal data

$\mathrm{C}_{8} \mathrm{H}_{8} \mathrm{~S}_{6}$

$M_{r}=296.50$

Monoclinic, $C 2 / c$

Hall symbol: -C $2 \mathrm{yc}$

$a=19.368$ (11) $\AA$

$b=7.703(4) \AA$

$c=17.150(8) \AA$

$\beta=108.59(2)^{\circ}$

$V=2425(2) \AA^{3}$

$Z=8$

$F(000)=1216$

$D_{\mathrm{x}}=1.624 \mathrm{Mg} \mathrm{m}^{-3}$

Mo $K \alpha$ radiation, $\lambda=0.71073 \AA$

Cell parameters from 9674 reflections

$\theta=3.3-27.6^{\circ}$

$\mu=1.09 \mathrm{~mm}^{-1}$

$T=291 \mathrm{~K}$

Block, yellow

$0.12 \times 0.10 \times 0.09 \mathrm{~mm}$

\section{Data collection}

Rigaku R-AXIS RAPID

diffractometer

Radiation source: fine-focus sealed tube

Graphite monochromator

$\omega$ scans

Absorption correction: multi-scan

(ABSCOR; Higashi, 1995)

$T_{\min }=0.881, T_{\max }=0.909$

11301 measured reflections

2773 independent reflections

2480 reflections with $I>2 \sigma(I)$

$R_{\text {int }}=0.025$

$\theta_{\text {max }}=27.5^{\circ}, \theta_{\min }=3.3^{\circ}$

$h=-25 \rightarrow 25$

$k=-10 \rightarrow 9$

$l=-21 \rightarrow 22$

\section{Refinement}

Refinement on $F^{2}$

Least-squares matrix: full

$R\left[F^{2}>2 \sigma\left(F^{2}\right)\right]=0.031$

$w R\left(F^{2}\right)=0.083$

$S=1.03$

2773 reflections

129 parameters

0 restraints

Primary atom site location: structure-invariant

direct methods

Secondary atom site location: difference Fourier map

Hydrogen site location: inferred from

neighbouring sites

$\mathrm{H}$-atom parameters constrained

$w=1 /\left[\sigma^{2}\left(F_{0}^{2}\right)+(0.043 P)^{2}+2.0438 P\right]$

where $P=\left(F_{\mathrm{o}}^{2}+2 F_{\mathrm{c}}^{2}\right) / 3$

$(\Delta / \sigma)_{\max }=0.006$

$\Delta \rho_{\max }=0.58$ e $\AA^{-3}$

$\Delta \rho_{\min }=-0.66$ e $\AA^{-3}$

Special details

Experimental. (See detailed section in the paper)

Geometry. All e.s.d.'s (except the e.s.d. in the dihedral angle between two 1.s. planes) are estimated using the full covariance matrix. The cell e.s.d.'s are taken into account individually in the estimation of e.s.d.'s in distances, angles and torsion angles; correlations between e.s.d.'s in cell parameters are only used when they are defined by crystal symmetry. An approximate (isotropic) treatment of cell e.s.d.'s is used for estimating e.s.d.'s involving 1.s. planes.

Refinement. Refinement of $F^{2}$ against ALL reflections. The weighted $R$-factor $w R$ and goodness of fit $S$ are based on $F^{2}$, conventional $R$-factors $R$ are based on $F$, with $F$ set to zero for negative $F^{2}$. The threshold expression of $F^{2}>\sigma\left(F^{2}\right)$ is used only for calculating $R$-factors(gt) $e t c$. and is not relevant to the choice of reflections for refinement. $R$-factors based on $F^{2}$ are statistically about twice as large as those based on $F$, and $R$ - factors based on ALL data will be even larger.

Fractional atomic coordinates and isotropic or equivalent isotropic displacement parameters $\left(\AA^{2}\right)$

\begin{tabular}{lllll}
\hline & $x$ & $y$ & $z$ & $U_{\text {iso }} * / U_{\text {eq }}$ \\
\hline C1 & $0.02208(11)$ & $0.7309(3)$ & $1.15260(11)$ & $0.0490(5)$ \\
H1 & -0.0004 & 0.7911 & 1.1849 & $0.059 *$ \\
C2 & $0.06543(12)$ & $0.5980(3)$ & $1.18273(11)$ & $0.0494(5)$
\end{tabular}




$\begin{array}{lllll}\text { H2 } & 0.0744 & 0.5615 & 1.2368 & 0.059^{*} \\ \text { C3 } & 0.07086(9) & 0.6402(2) & 1.03633(10) & 0.0331(3) \\ \text { C4 } & 0.09038(9) & 0.6398(2) & 0.96816(10) & 0.0348(3) \\ \text { C5 } & 0.12943(10) & 0.7609(2) & 0.84888(10) & 0.0375(4) \\ \text { C6 } & 0.17548(9) & 0.6286(2) & 0.87949(10) & 0.0381(4) \\ \text { C7 } & 0.18062(13) & 1.0834(3) & 0.83038(14) & 0.0552(5) \\ \text { H7A } & 0.1538 & 1.1248 & 0.8651 & 0.083^{*} \\ \text { H7B } & 0.1850 & 1.1747 & 0.7941 & 0.083^{*} \\ \text { H7C } & 0.2283 & 1.0472 & 0.8638 & 0.083^{*} \\ \text { C8 } & 0.30945(12) & 0.4634(3) & 0.92717(15) & 0.0608(6) \\ \text { H8A } & 0.3209 & 0.5384 & 0.9743 & 0.091^{*} \\ \text { H8B } & 0.3531 & 0.4385 & 0.9141 & 0.091^{*} \\ \text { H8C } & 0.2890 & 0.3571 & 0.9391 & 0.091^{*} \\ \text { S1 } & 0.00813(3) & 0.79005(6) & 1.05124(3) & 0.04253(13) \\ \text { S2 } & 0.10518(3) & 0.49359(6) & 1.11799(3) & 0.04402(13) \\ \text { S3 } & 0.05498(2) & 0.78415(6) & 0.88578(3) & 0.04151(13) \\ \text { S4 } & 0.15503(3) & 0.49566(6) & 0.95261(3) & 0.04458(14) \\ \text { S5 } & 0.13348(3) & 0.90281(7) & 0.77079(3) & 0.05321(15) \\ \text { S6 } & 0.24524(3) & 0.56800(10) & 0.84168(4) & 0.06234(18)\end{array}$

Atomic displacement parameters $\left(\AA^{2}\right)$

\begin{tabular}{lllllll}
\hline & $U^{11}$ & $U^{22}$ & $U^{33}$ & $U^{12}$ & $U^{13}$ & $U^{23}$ \\
\hline C1 & $0.0601(12)$ & $0.0557(12)$ & $0.0353(9)$ & $0.0035(9)$ & $0.0211(8)$ & $-0.0054(8)$ \\
C2 & $0.0591(11)$ & $0.0599(13)$ & $0.0293(9)$ & $0.0019(10)$ & $0.0142(8)$ & $0.0037(8)$ \\
C3 & $0.0323(7)$ & $0.0330(8)$ & $0.0354(8)$ & $0.0023(6)$ & $0.0127(6)$ & $0.0048(6)$ \\
C4 & $0.0353(8)$ & $0.0347(9)$ & $0.0377(8)$ & $0.0030(6)$ & $0.0160(7)$ & $0.0063(6)$ \\
C5 & $0.0468(9)$ & $0.0376(9)$ & $0.0311(8)$ & $-0.0072(7)$ & $0.0166(7)$ & $0.0002(6)$ \\
C6 & $0.0405(8)$ & $0.0442(10)$ & $0.0343(8)$ & $-0.0055(7)$ & $0.0185(7)$ & $-0.0019(7)$ \\
C7 & $0.0602(12)$ & $0.0458(12)$ & $0.0593(13)$ & $-0.0100(10)$ & $0.0187(10)$ & $0.0034(9)$ \\
C8 & $0.0425(11)$ & $0.0773(16)$ & $0.0651(14)$ & $0.0057(10)$ & $0.0206(10)$ & $-0.0037(12)$ \\
S1 & $0.0492(3)$ & $0.0424(3)$ & $0.0420(2)$ & $0.01172(19)$ & $0.0229(2)$ & $0.00695(18)$ \\
S2 & $0.0462(3)$ & $0.0476(3)$ & $0.0400(2)$ & $0.01051(19)$ & $0.0161(2)$ & $0.01361(18)$ \\
S3 & $0.0447(2)$ & $0.0423(3)$ & $0.0404(2)$ & $0.00839(19)$ & $0.01765(19)$ & $0.01208(18)$ \\
S4 & $0.0482(3)$ & $0.0449(3)$ & $0.0505(3)$ & $0.0142(2)$ & $0.0295(2)$ & $0.01503(19)$ \\
S5 & $0.0789(4)$ & $0.0510(3)$ & $0.0320(2)$ & $-0.0161(3)$ & $0.0210(2)$ & $0.00457(19)$ \\
S6 & $0.0543(3)$ & $0.0919(5)$ & $0.0539(3)$ & $0.0106(3)$ & $0.0356(3)$ & $0.0085(3)$ \\
& & & & & &
\end{tabular}

Geometric parameters ( $\left.\AA,{ }^{\circ}\right)$

\begin{tabular}{llll}
\hline $\mathrm{C} 1-\mathrm{C} 2$ & $1.321(3)$ & $\mathrm{C} 5-\mathrm{S} 3$ & $1.759(2)$ \\
$\mathrm{C} 1-\mathrm{S} 1$ & $1.733(2)$ & $\mathrm{C} 6-\mathrm{S} 6$ & $1.7386(19)$ \\
$\mathrm{C} 1-\mathrm{H} 1$ & 0.9300 & $\mathrm{C} 6-\mathrm{S} 4$ & $1.7591(18)$ \\
$\mathrm{C} 2-\mathrm{S} 2$ & $1.737(2)$ & $\mathrm{C} 7-\mathrm{S} 5$ & $1.794(2)$ \\
$\mathrm{C} 2-\mathrm{H} 2$ & 0.9300 & $\mathrm{C} 7-\mathrm{H} 7 \mathrm{~A}$ & 0.9600 \\
$\mathrm{C} 3-\mathrm{C} 4$ & $1.339(2)$ & $\mathrm{C} 7-\mathrm{H} 7 \mathrm{~B}$ & 0.9600 \\
$\mathrm{C} 3-\mathrm{S} 1$ & $1.7525(18)$ & $\mathrm{C} 7-\mathrm{H} 7 \mathrm{C}$ & 0.9600 \\
$\mathrm{C} 3-\mathrm{S} 2$ & $1.7565(17)$ & $\mathrm{C} 8-\mathrm{S} 6$ & $1.784(2)$
\end{tabular}




$\begin{array}{llll}\mathrm{C} 4-\mathrm{S} 3 & 1.7565(18) & \mathrm{C} 8-\mathrm{H} 8 \mathrm{~A} & 0.9600 \\ \mathrm{C} 4-\mathrm{S} 4 & 1.7562(18) & \mathrm{C} 8-\mathrm{H} 8 \mathrm{~B} & 0.9600 \\ \mathrm{C} 5-\mathrm{C} 6 & 1.346(3) & \mathrm{C} 8-\mathrm{H} 8 \mathrm{C} & 0.9600 \\ \mathrm{C} 5-\mathrm{S} 5 & 1.7498(18) & & \\ & & & 109.5 \\ \mathrm{C} 2-\mathrm{C} 1-\mathrm{S} 1 & 118.21(15) & \mathrm{S} 5-\mathrm{C} 7-\mathrm{H} 7 \mathrm{~A} & 109.5 \\ \mathrm{C} 2-\mathrm{C} 1-\mathrm{H} 1 & 120.9 & \mathrm{~S} 5-\mathrm{C} 7-\mathrm{H} 7 \mathrm{~B} & 109.5 \\ \mathrm{~S} 1-\mathrm{C} 1-\mathrm{H} 1 & 120.9 & \mathrm{H} 7 \mathrm{~A}-\mathrm{C} 7-\mathrm{H} 7 \mathrm{~B} & 109.5 \\ \mathrm{C} 1-\mathrm{C} 2-\mathrm{S} 2 & 117.70(15) & \mathrm{S} 5-\mathrm{C} 7-\mathrm{H} 7 \mathrm{C} & 109.5 \\ \mathrm{C} 1-\mathrm{C} 2-\mathrm{H} 2 & 121.1 & \mathrm{H} 7 \mathrm{~A}-\mathrm{C} 7-\mathrm{H} 7 \mathrm{C} & 109.5 \\ \mathrm{~S} 2-\mathrm{C} 2-\mathrm{H} 2 & 121.1 & \mathrm{H} 7 \mathrm{~B}-\mathrm{C} 7-\mathrm{H} 7 \mathrm{C} & 109.5 \\ \mathrm{C} 4-\mathrm{C} 3-\mathrm{S} 1 & 122.09(13) & \mathrm{S} 6-\mathrm{C} 8-\mathrm{H} 8 \mathrm{~A} & 109.5 \\ \mathrm{C} 4-\mathrm{C} 3-\mathrm{S} 2 & 123.66(14) & \mathrm{S} 6-\mathrm{C} 8-\mathrm{H} 8 \mathrm{~B} & 109.5 \\ \mathrm{~S} 1-\mathrm{C} 3-\mathrm{S} 2 & 114.26(9) & \mathrm{H} 8 \mathrm{~A}-\mathrm{C} 8-\mathrm{H} 8 \mathrm{~B} & 109.5 \\ \mathrm{C} 3-\mathrm{C} 4-\mathrm{S} 3 & 123.67(14) & \mathrm{S} 6-\mathrm{C} 8-\mathrm{H} 8 \mathrm{C} & 109.5 \\ \mathrm{C} 3-\mathrm{C} 4-\mathrm{S} 4 & 123.23(13) & \mathrm{H} 8 \mathrm{~A}-\mathrm{C} 8-\mathrm{H} 8 \mathrm{C} & 109.5 \\ \mathrm{~S} 3-\mathrm{C} 4-\mathrm{S} 4 & 113.10(10) & \mathrm{H} 8 \mathrm{~B}-\mathrm{C} 8-\mathrm{H} 8 \mathrm{C} & 94.57(9) \\ \mathrm{C} 6-\mathrm{C} 5-\mathrm{S} 5 & 125.86(14) & \mathrm{C} 1-\mathrm{S} 1-\mathrm{C} 3 & 94.58(10) \\ \mathrm{C} 6-\mathrm{C} 5-\mathrm{S} 3 & 117.01(13) & \mathrm{C} 2-\mathrm{S} 2-\mathrm{C} 3 & 94.04(8) \\ \text { S5-C5-S3 } & 116.99(11) & \mathrm{C} 4-\mathrm{S} 3-\mathrm{C} 5 & 94.24(9) \\ \text { C5-C6-S6 } & 123.71(14) & \mathrm{C} 4-\mathrm{S} 4-\mathrm{C} 6 & 100.79(10) \\ \text { C5-C6-S4 } & 116.55(14) & \mathrm{C} 5-\mathrm{S} 5-\mathrm{C} 7 & 103.63(10) \\ \text { S6-C6-S4 } & 119.21(11) & \mathrm{C} 6-\mathrm{S} 6-\mathrm{C} 8 & \end{array}$

